\title{
Radiological Assessment of Paediatric Fungal Infections: A Pictorial Review With Focus on PET/MRI
}

\author{
ALESSIA VAROTTO ${ }^{1}$, GIOVANNA ORSATTI ${ }^{1}$, FILIPPO CRIMİ ${ }^{1}$, DIEGO CECCHIN ${ }^{2}$, \\ TIZIANA TOFFOLUTTI ${ }^{1}$, PIETRO ZUCCHETTA ${ }^{2}$ and ROBERTO STRAMARE ${ }^{1}$ \\ ${ }^{1}$ Radiology Unit, Department of Medicine DIMED, University-Hospital Padua, Padua, Italy; \\ ${ }^{2}$ Nuclear Medicine Unit, Department of Medicine DIMED, University-Hospital Padua, Padua, Italy
}

\begin{abstract}
Paediatric invasive fungal infections have significantly increased over the past few decades, in particular among the immunocompromised population. Candida and Aspergillus spp. are still the most commonly isolated organisms. Image-based assessment of fungal infections can indeed be challenging especially in oncological patients where the differential diagnosis relative to other infections and neoplastic lesions cannot be often obvious. Therefore, the knowledge of the main radiological features associated with fungal infections is crucial to achieve an early correct diagnosis and address the most appropriate therapeutic approach. Thus, our aim was to review the main radiological features of paediatric fungal infections with particular focus on positron emission tomography/magnetic resonance imaging (PET/MRI), referring to the experience of our tertiary level hospital.
\end{abstract}

Invasive fungal disease (IFD) can be a life-threatening condition, in particular in paediatric patients who undergo chemotherapeutic or immunosuppressive treatments, in children with congenital or acquired immunodeficiencies, in premature neonates and in patients with fixed prostheses or catheters. IFD incidence has increased over the past few decades among the paediatric population, due to the improvement of oncological therapies which have significantly increased the survival but lead to an inevitable weakening of immune response. The most frequent

This article is freely accessible online.

Correspondence to: Filippo Crimì, Radiology Unit, Department of Medicine-DIMED, University-Hospital of Padua, via Giustiniani 2, Padua, 35128, Italy. E-mail: crimifilippo@gmail.com

Key Words: Paediatric infections; invasive fungal infection, $\mathrm{PET} / \mathrm{MR}, \mathrm{CT}$, MRI, review. pathogens involved are Candida albicans/non-albicans spp, Aspergillus spp and Cryptococcus neoformans, Histoplasma capsulatum, Zygomycetes, Fusarium, and other pathogens (1, 2). Fungal infections have a significant impact on patients' outcome, with a mortality rate of about $15.8 \%$ for children affected by candidemia and about $18 \%$ for children with invasive aspergillosis (3). Therefore, an early diagnosis is essential to assure a prompt treatment. Since clinical manifestations are often non-specific, the radiological examination plays a key role for the detection of IFD and for the assessment of the disease extent. In addition to conventional imaging [ultrasound (US), plain X ray films, computed tomography (CT) and magnetic resonance imaging (MRI)], in the last years an increasing interest has been developed in hybrid whole-body imaging techniques [i.e. positron emission tomography/CT (PET/CT), PET/MRI], in particular in PET/MRI which combines the benefits of high soft tissue contrast resolution of MR with the metabolic information of PET, providing also the intrinsic advantage of a low dose radiation exposure. Therefore, integrated, simultaneously acquired PET/MRI can be an innovative and highly promising diagnostic tool for the evaluation of systemic fungal infections in paediatric patients. Nevertheless, the differential diagnosis with other infections and with malignant lesions can be challenging and histopathological confirmation is still strongly required when feasible to provide a definite diagnosis.

\section{PET/CT and the Potential Role of PET/MRI}

Whole-body hybrid imaging techniques such as $18 \mathrm{~F}$-labeled fluoro-2-deoxyglucose ( ${ }^{18}$ F-FDG)-PET/CT and PET/MRI can allow an early diagnosis of fungal infection foci mainly because of the high glucose consumption of these lesions, also providing a morphological definition of the disease extent. Moreover, the combination of the metabolic and the morphological information can be crucial in guiding the 


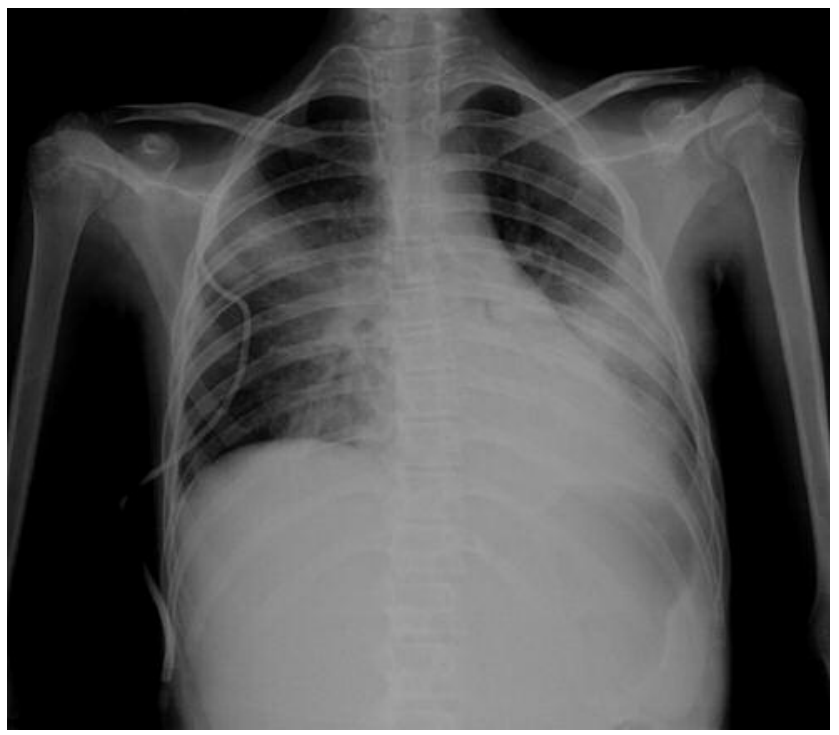

Figure 1. Chest X-ray showing bilateral lung parenchymal consolidations and left pleural effusion in a 12-year-old girl, with pulmonary aspergillosis.

surgeons to choose the best target for a biopsy and to assess the response to antifungal treatment, especially if no morphological modifications are seen on conventional imaging. Nevertheless, the characterization of hypermetabolic areas can be difficult when using PET/CT because of the low contrast resolution especially in case of soft tissues involvement and besides PET/CT exposes children to a higher dose of radiation compared to CT alone MRI. Therefore, in the last few years, an increasing interest has been developed towards hybrid PET/MRI because of its potential to overcome these limits (4).

\section{Lung}

The lungs are the most commonly affected site in IFD and fungal pulmonary involvement is associated with high mortality. Aspergillus is the most common pathogen found in fungal pulmonary infections, in particular in haematological malignancies. The clinical manifestations include fever, cough, dyspnea and haemoptysis (5). Chest Xray examination is the first-choice imaging modality but it often shows non-specific signs, such as ill-defined parenchymal opacities usually in both lungs and pleural effusion (Figure 1); sometimes well-defined and cavitated nodules can be evident (Figure 2).

The radiological standard of reference in pulmonary fungal involvement is the chest CT scan; common findings in invasive asperigillosis are the presence of multiple bilateral nodules usually surrounded by ground glass opacity (halo sign) (Figure 3) and wedge-shaped areas of

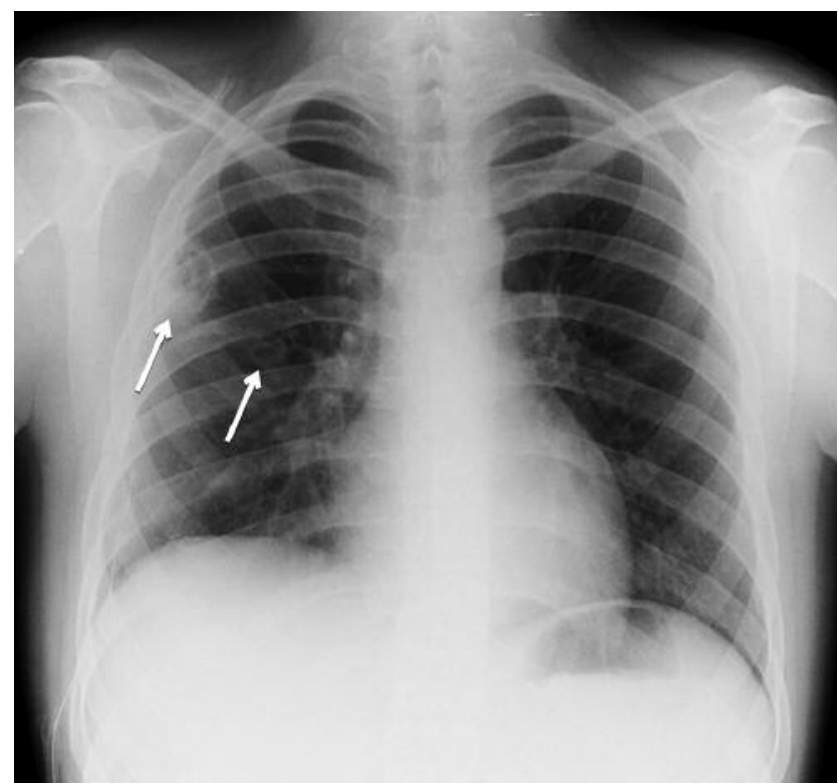

Figure 2. Chest $X$-ray demonstrating two cavitated lesions (arrows) in the right lung in a 16-year-old boy with pulmonary aspergillosis.

consolidation corresponding to haemorrhagic infarcts. The air-crescent sign, due to the central necrotic area inside a consolidation, can also be seen in some cases of aspergillosis during recovery (Figure 4). In candidiasis CT scan, bilateral pulmonary consolidations with possible cavitation and nodules can be found as well (Figure 5) (2).

The reverse halo sign instead, defined as the presence of ground glass attenuation surrounded by consolidation, can be more frequently seen in haematological patients with Mucor mycosis (2).

Lung visualization with MRI is definitely still a challenge, even though in recent years the introduction of lungdedicated gradient echo (GE) sequences with short echo time (TE) (such as VIBE, UTE) in PET/MRI protocols has improved the chances for the detection of pulmonary fungal involvement. Parenchymal consolidations and nodules can be seen as relatively high intensity signal lesions depending on their composition (proper solid lesions appear more hyperintense than ground glass lesions in low TE gradient echo sequences because of their higher density); excavations are usually seen as hypointense areas within (Figures 6 and 7). Furthermore, the combination with PET, adding the metabolic information, improves the detection of pulmonary fungal involvement in case of avid lesions and allows the assessment of treatment response. Indeed, the evidence of a decrease in the hypermetabolic activity of fungal foci after antifungal therapy helps to confirm the diagnosis especially when no morphological changes within the lesion are seen and demonstrates the efficacy of the treatment protocol (6). 


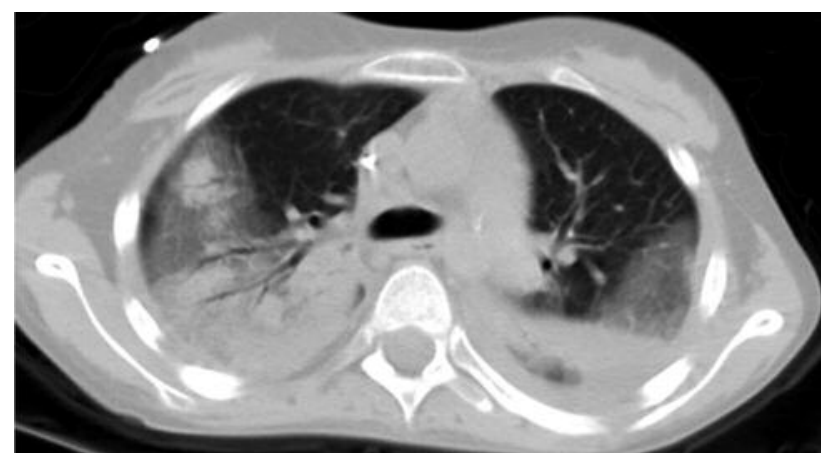

Figure 3. Chest CT scan illustrating bilateral parenchymal consolidations surrounded by ground glass opacities and pleural effusion (same patient shown in Figure 1).

\section{Nasal Cavities and Sinuses}

Nasal and paranasal fungal infections are quite common in immunocompromised paediatric patients with a mortality rate of approximately $50 \%$. The clinical signs and symptoms include fever, headache, facial pain and oedema, nasal congestion and epistasis.

Middle turbinate, ethmoid cells, maxillary and sphenoid sinuses are more frequently involved. The assessment of the disease extent is crucial in these sites especially because of the risk of CNS involvement. CT scan usually shows thickening of the nasal or paranasal mucosa, with infiltration of the periantral fat planes. In late disease, bone erosion with orbital invasion and intracranial extension can be seen. On MRI, in addition to the mucosal thickening and fat planes infiltration, focal loss of contrast enhancement can be appreciated after gadolinium injection in the infected sino-nasal mucosa (Figure 8). The functional information given by PET can improve the diagnostic accuracy of MRI and also provides a valuable tool for treatment response evaluation detecting a potential FDG uptake reduction or disappearance (7-9).

\section{Central Nervous System}

Fungal infections affecting the central nervous system have a very poor prognosis with high mortality, especially in immunocompromised patients (almost 100\%). The brain is the third most common site of fungal infection in patients with haematological malignancies after lungs and paranasal sinuses. Clinical symptoms are non-specific (confusion, hemiparesis, dysarthria, lethargy, seizures, possible fever) but their presence in immunocompromised children should suggest the diagnosis. Two main patterns can be present: focal lesions or diffuse meningoencephalitis. Fungal brain focal lesions are more common in Candida and Aspergillus infections; they are

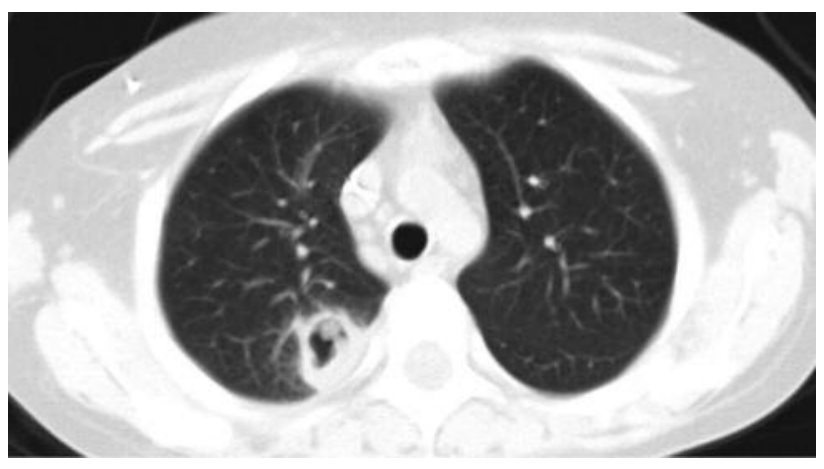

Figure 4. A lesion in the right lung showing a crescent-shaped air content ("air crescent sign") on the chest CT scan in a 11-year-old boy with aspergillosis.

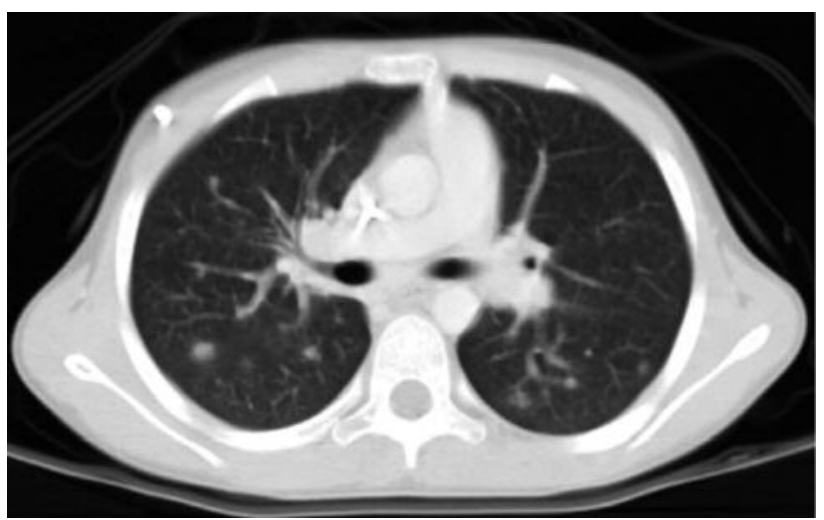

Figure 5. Chest CT scan showing multiple bilateral lung nodules in a 9-year-old boy with disseminated Candida.

frequently multiple and usually involve the basal ganglia. CT findings are non-specific for fungal infection, such as focal hypodensities with a peripheral rim of contrast enhancement and often surrounded by oedema. On MRI, fungal abscesses show a central core, hypointense on T1-weighted and hyperintense on T2-weighted sequences, while the peripheral rim is iso-hyperintense on T1-weighted and hypointense on T2weighted images. Peripheral enhancement after gadolinium injection and signal restriction on diffusion-weighted images (DWI) usually help to characterize the lesions (Figure 9). Fungal meningoencephalitis is most frequently caused by Cryptococcus, followed by Aspergillus and Candida. In encephalitis, CT scan of the brain may show ill-defined hypodense lesions with areas of increased attenuation, due to haemorrhage or increased metal ions. On MRI examinations, the infected areas are iso-hypointense on T1-weighted, hyperintense on T2-weighted/FLAIR images and with signal 

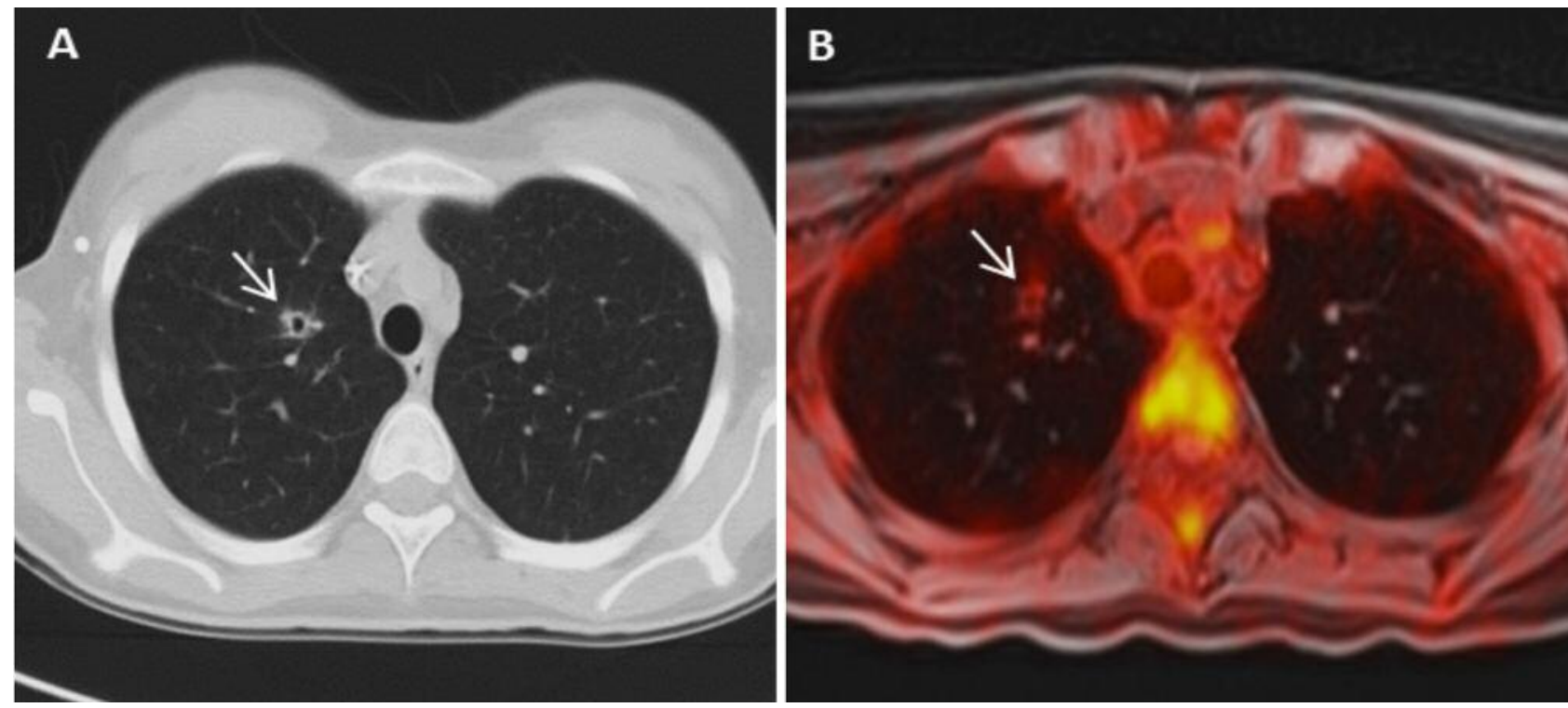

Figure 6. A lung cavitated nodule (arrow) on CT scan (A) and on fused PET/MRI (B), showing a slight peripheral hypermetabolism, in a 13-yearold girl with pulmonary aspergillosis.

restriction on DWI. Micro-haemorrhages and focal deposits of metals are usually found within the lesions as decreased signal intensity on T2-weighted sequences. In fungal meningitis, radiological examinations show meningeal thickening and enhancement, both on CT and MRI, but the demonstration of fungi in CSF is necessary for the diagnosis. On PET, the visualization of lesions showing FDG uptake may be difficult due to the high intrinsic hypermetabolism of the cerebral cortex. Nevertheless, in case of fungal abscesses, especially when localized in the white matter, the combination of PET and MRI might help their detection, and again giving information about their response to treatment (Figures 10 and 11) $(10,11)$.

\section{Liver and Spleen}

Liver and spleen can be involved in paediatric IFD, in particular in children with haematological malignancies. Candida species are the most frequent pathogens affecting these organs with a mortality rate up to $30 \%$ (12). US can show four different patterns of fungal infection: an hypoechoic necrotic lesion surrounded by a hyperechoic rim of inflammatory cells and an external fibrotic hypoechoic ring, an inner hyperechoic nidus surrounded by a hypoechoic fibrotic ring; hypoechoic lesion that is expression of fibrotic changes after inflammation; hyperechoic scar or calcification (13). CT scan usually shows rounded hypodense lesions, sometimes with a hyperdense core. These nodules can demonstrate contrast enhancement of the whole lesion or an enhanced peripheral rim (14). On MRI, these lesions appear slightly hypointense on T1w images and strongly

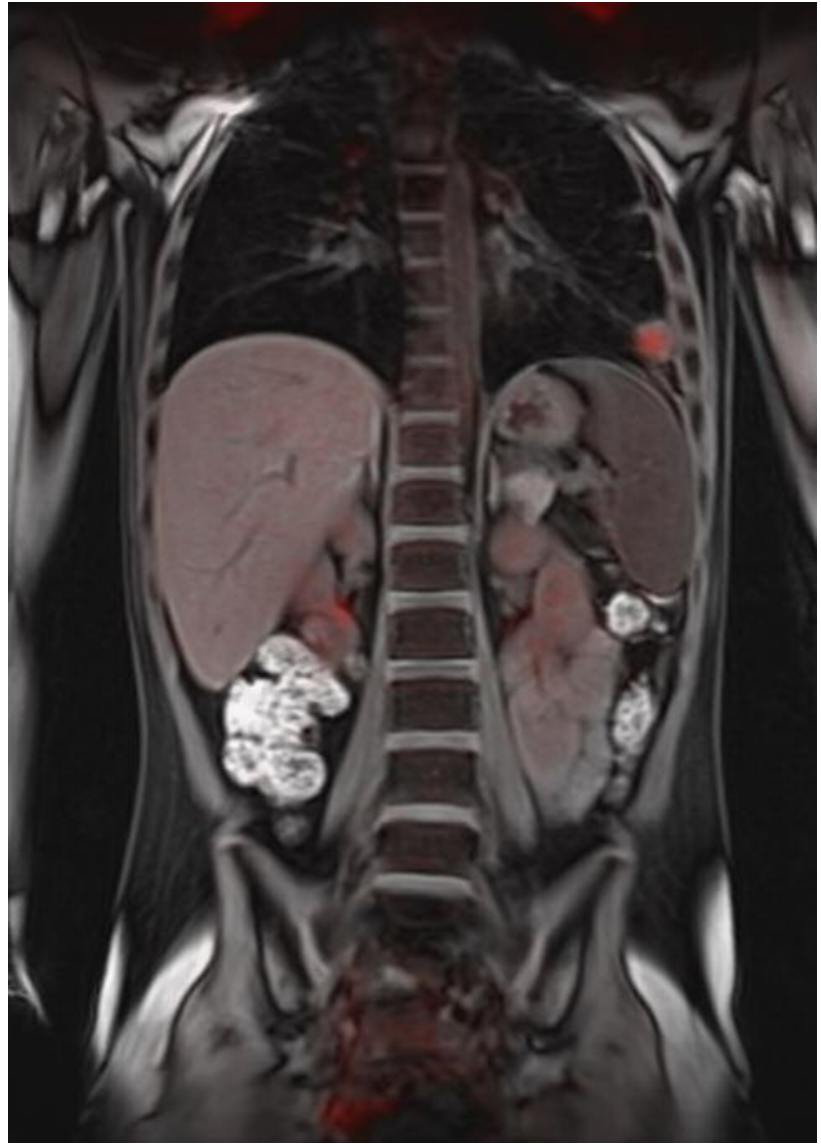

Figure 7. A hypermetabolic consolidation in the left lung (arrow) on fused PET/MR-VIBE image in a 12-year-old girl affected by Aspergillus. 

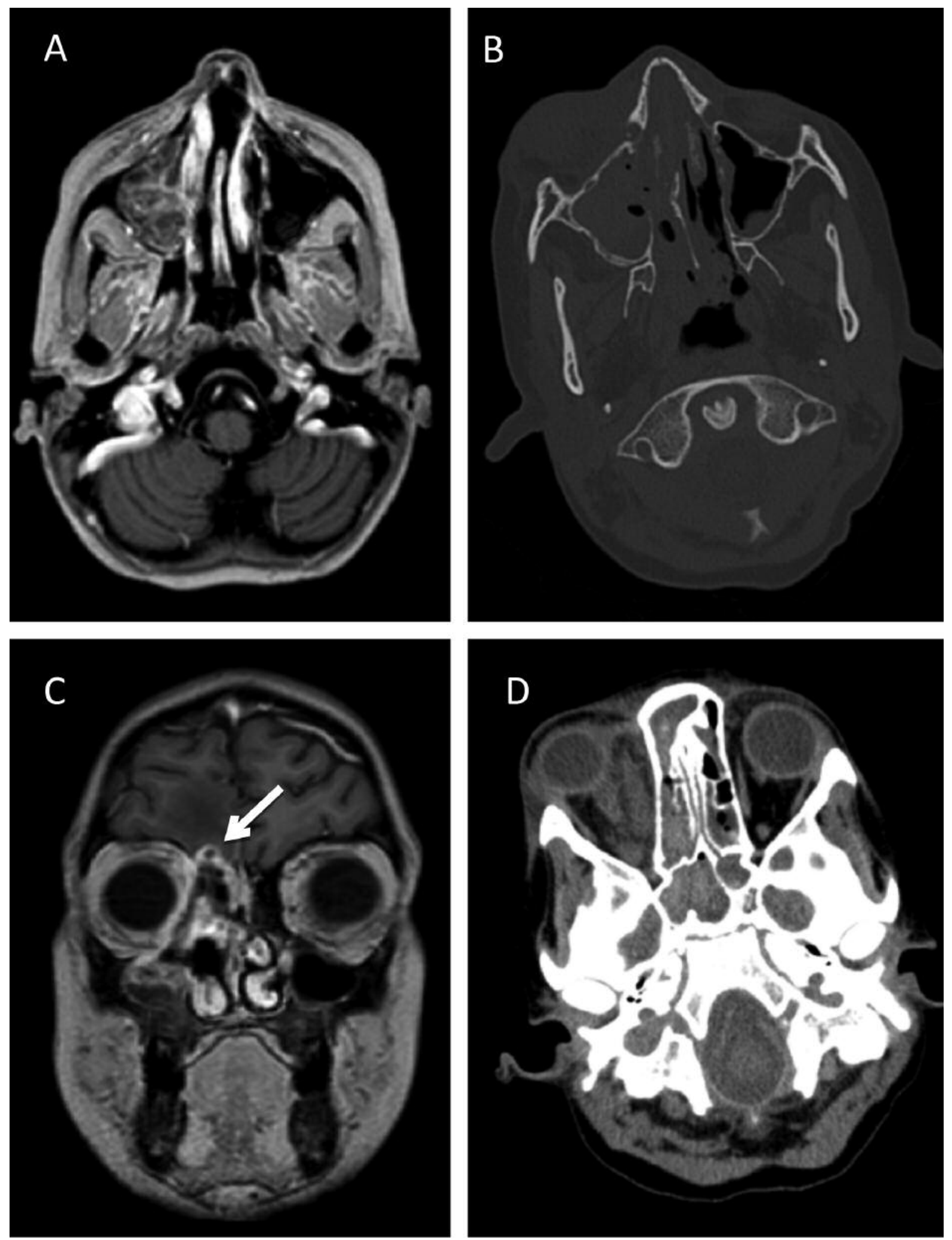

Figure 8. A 11-year-old boy affected by Mucor mycosis: a large mycotic lesion can be seen in the right maxillary sinus (A), causing diffuse bone erosion of the sinus medial wall $(B)$ and intrathecal spread of the fungal infection (arrow in $C$ ) surrounded by oedema. Orbital cellulitis can be also appreciated $(D)$, involving the right medial rectus muscle. 

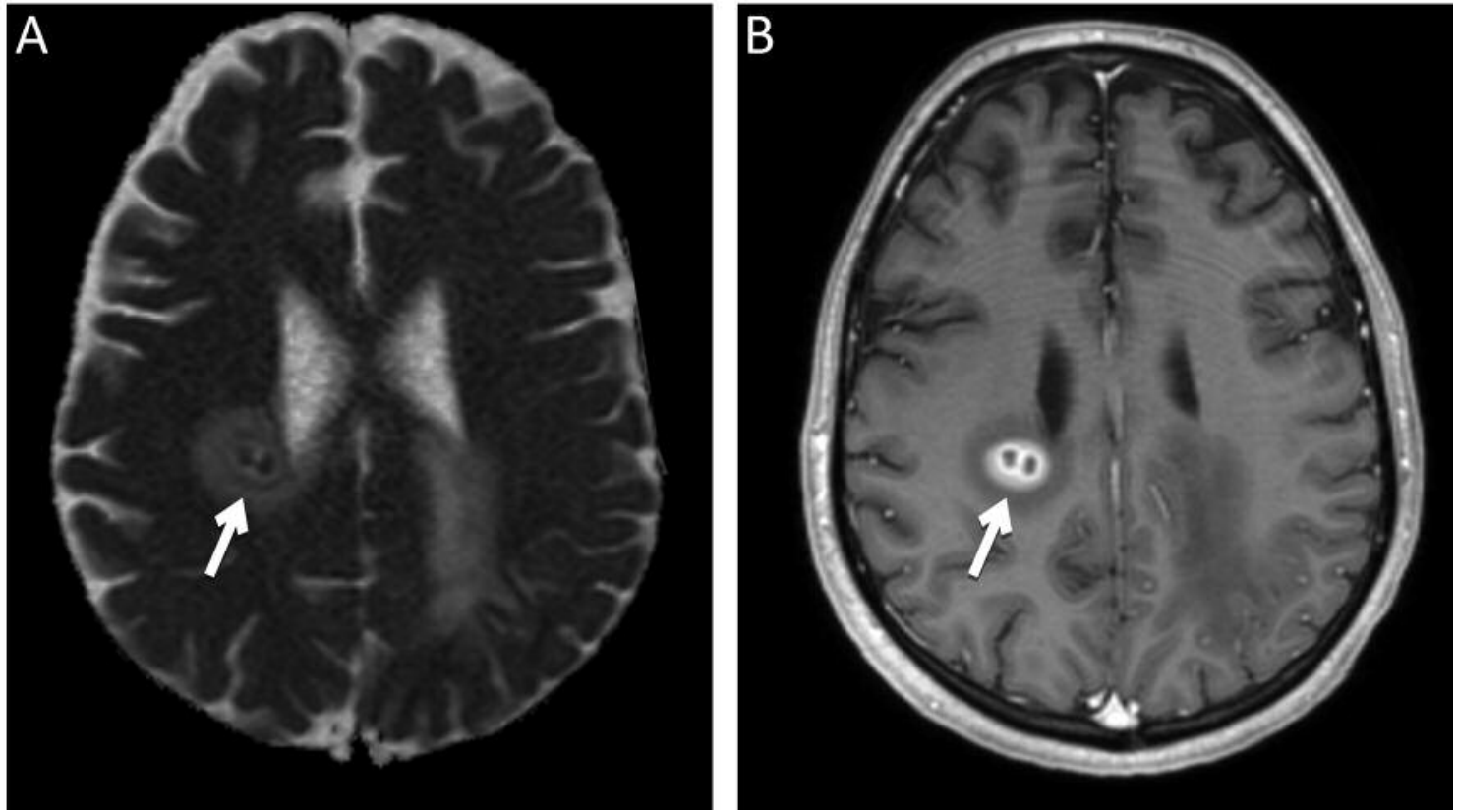

Figure 9. Fungal brain abscess (arrows) in a 16-year-old boy characterized by central restricted diffusion on ADC map (A) and peripheral rim of enhancement after Gadolinium (B).
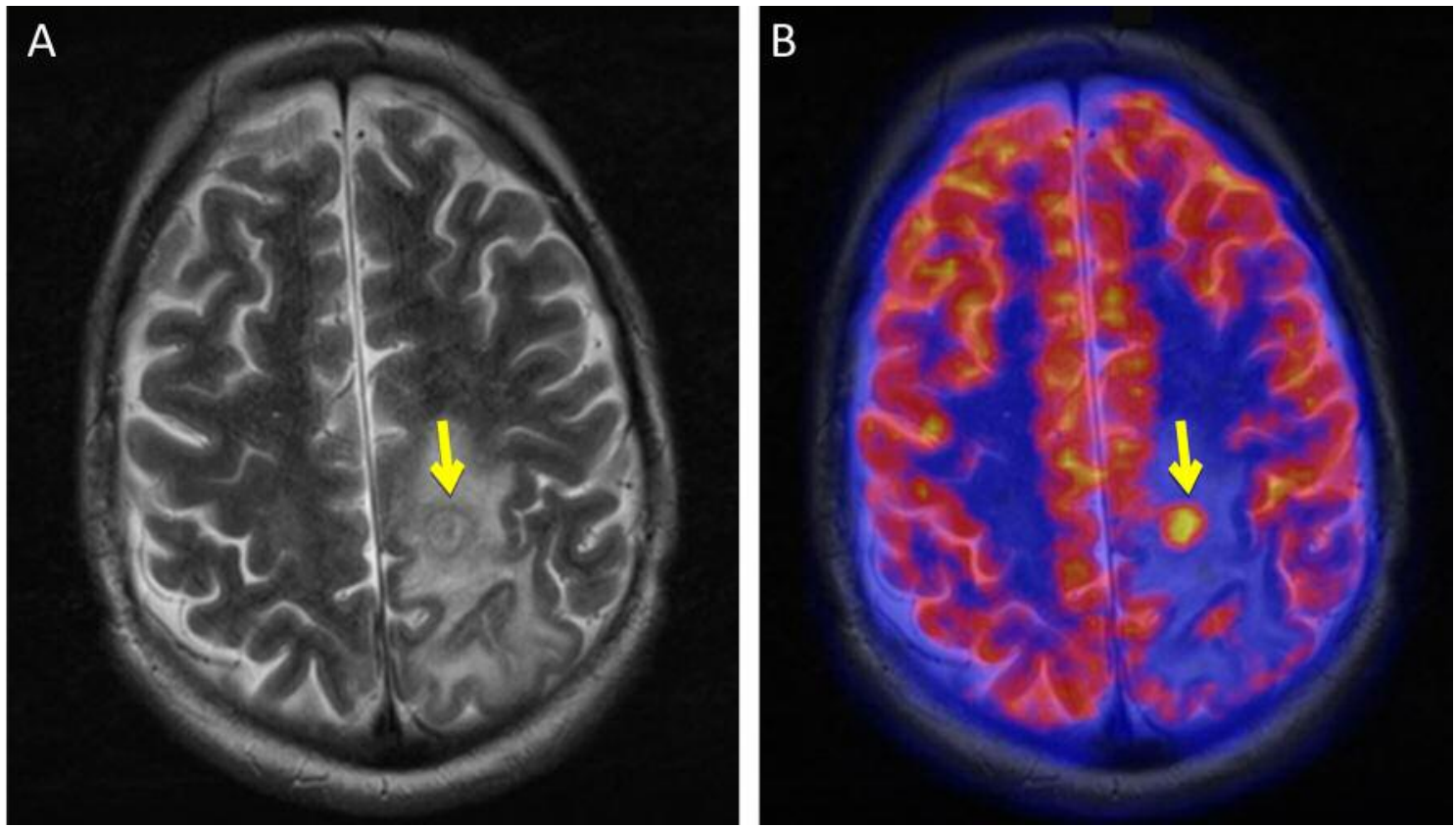

Figure 10. Candida cerebral abscess (arrows) characterized by surrounding oedema on T2-weighted image (A) and significant hypermetabolism on fused PET/MRI (B) in a 17-year-old boy.

hyperintense on $\mathrm{T} 2 \mathrm{w}$ sequences with an intense and early peripheral enhancement after gadolinium injection. Some studies have demonstrated that ${ }^{18}$ F-FDG-PET may be a feasible and sensitive tool for the detection of liver and splenic fungal lesions and the assessment of residual disease if compared with conventional imaging. Therefore, the integrated PET/MRI, especially in paediatric patients, can play an important role (15). 

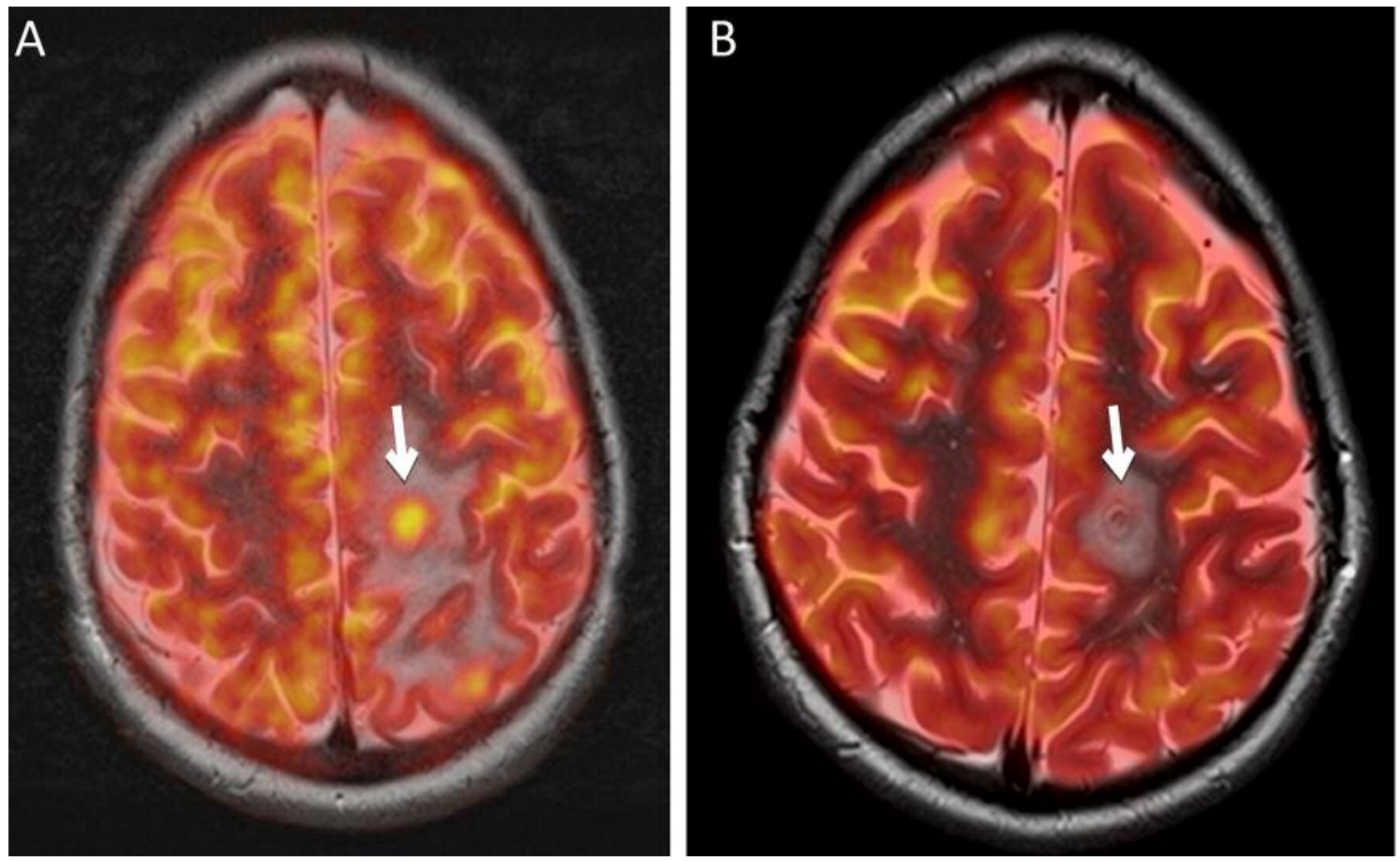

Figure 11. Fused PET/MR image of the same patient shown in Figure 10, at diagnosis (A) and after four weeks of anti-fungal treatment (B): the lesion demonstrates a slight reduction in size whereas its hypermetabolism is not appreciable anymore. A significant reduction of the surrounding oedema is also present.

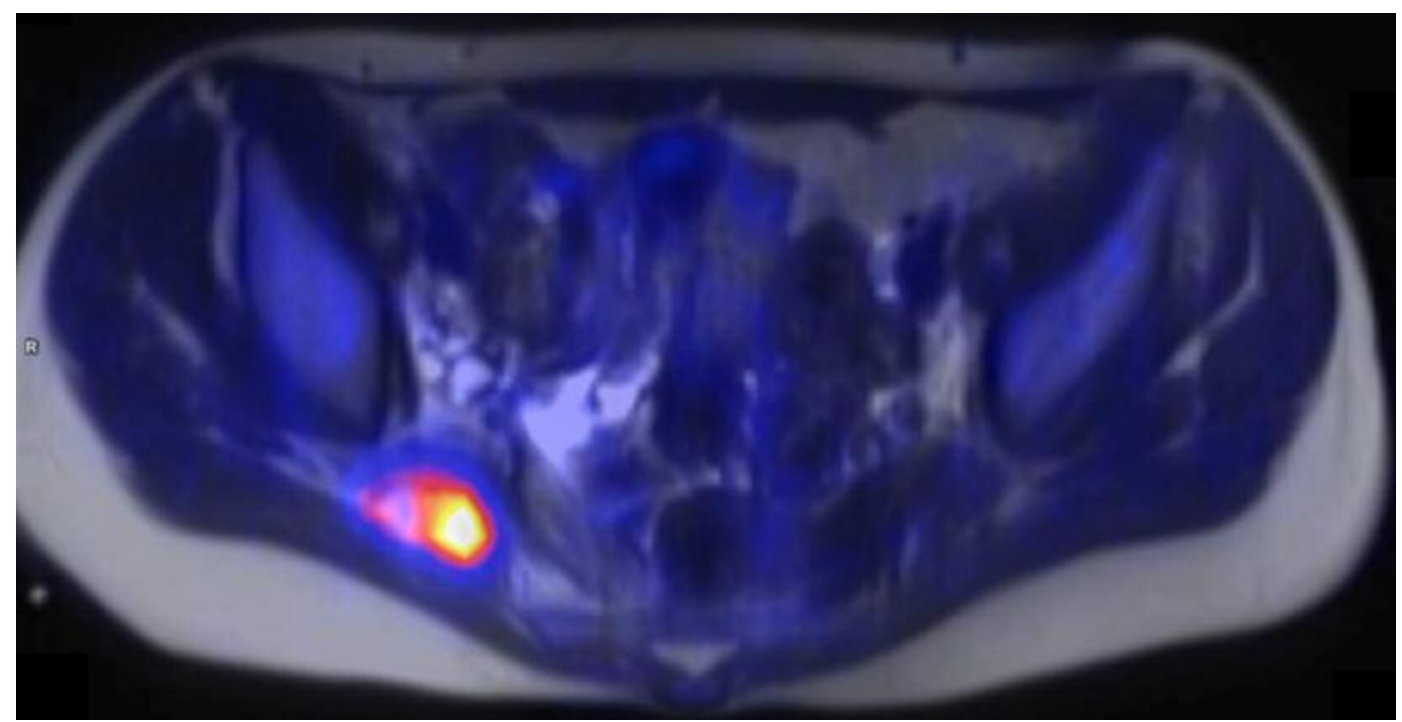

Figure 12. A hypermetabolic fungal abscess (arrow) in the right piriformis muscle on fused PET/MR image in a 16-year-old girl affected by Aspergillus fumigatus.

\section{Other Organs}

Rarely, other organs can be affected by IFD. The hematogenous spread of pathogens makes possible the localization of infection also in soft tissues (skin, subcutaneous tissue, muscles), bones, kidney, etc. In these cases, a whole-body scan with PET/MR can reasonably help the detection of uncommon sites of infection (Figures 12-14). 


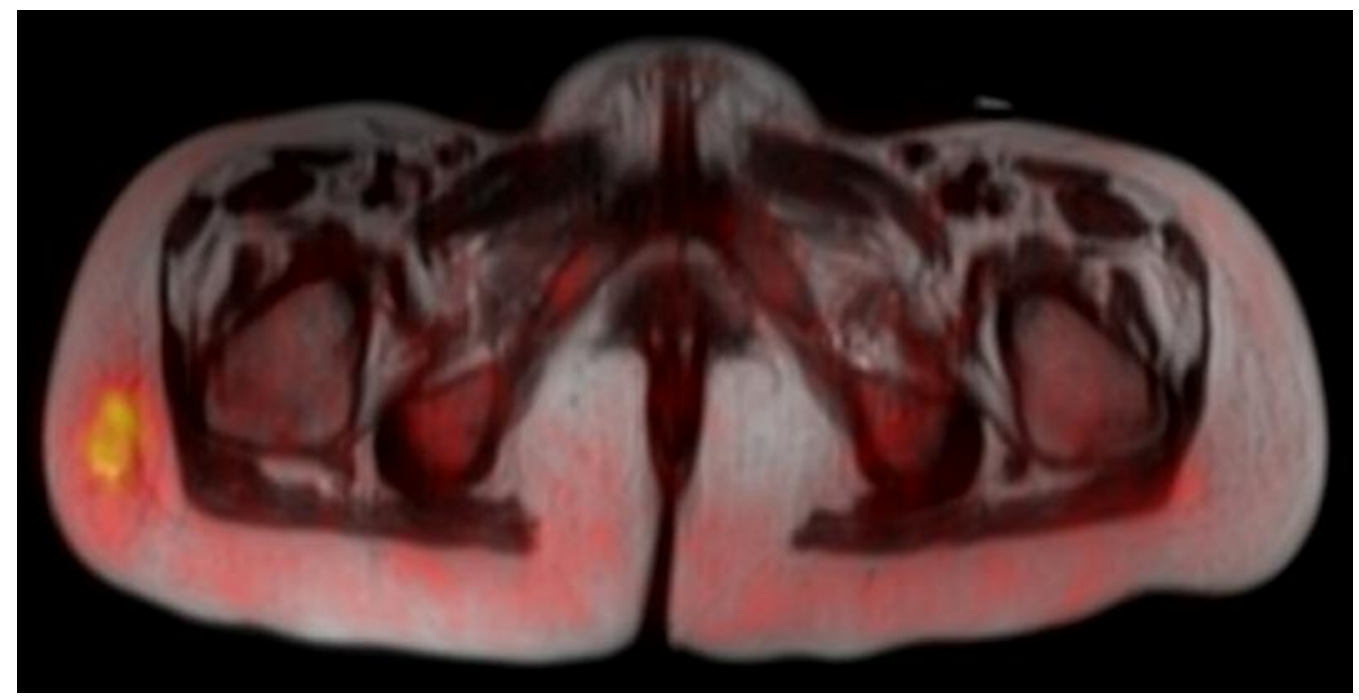

Figure 13. A hypermetabolic Aspergillus lesion in the subcutaneous tissue (arrow) on fused PET/MR image, in a 12-year-old girl.
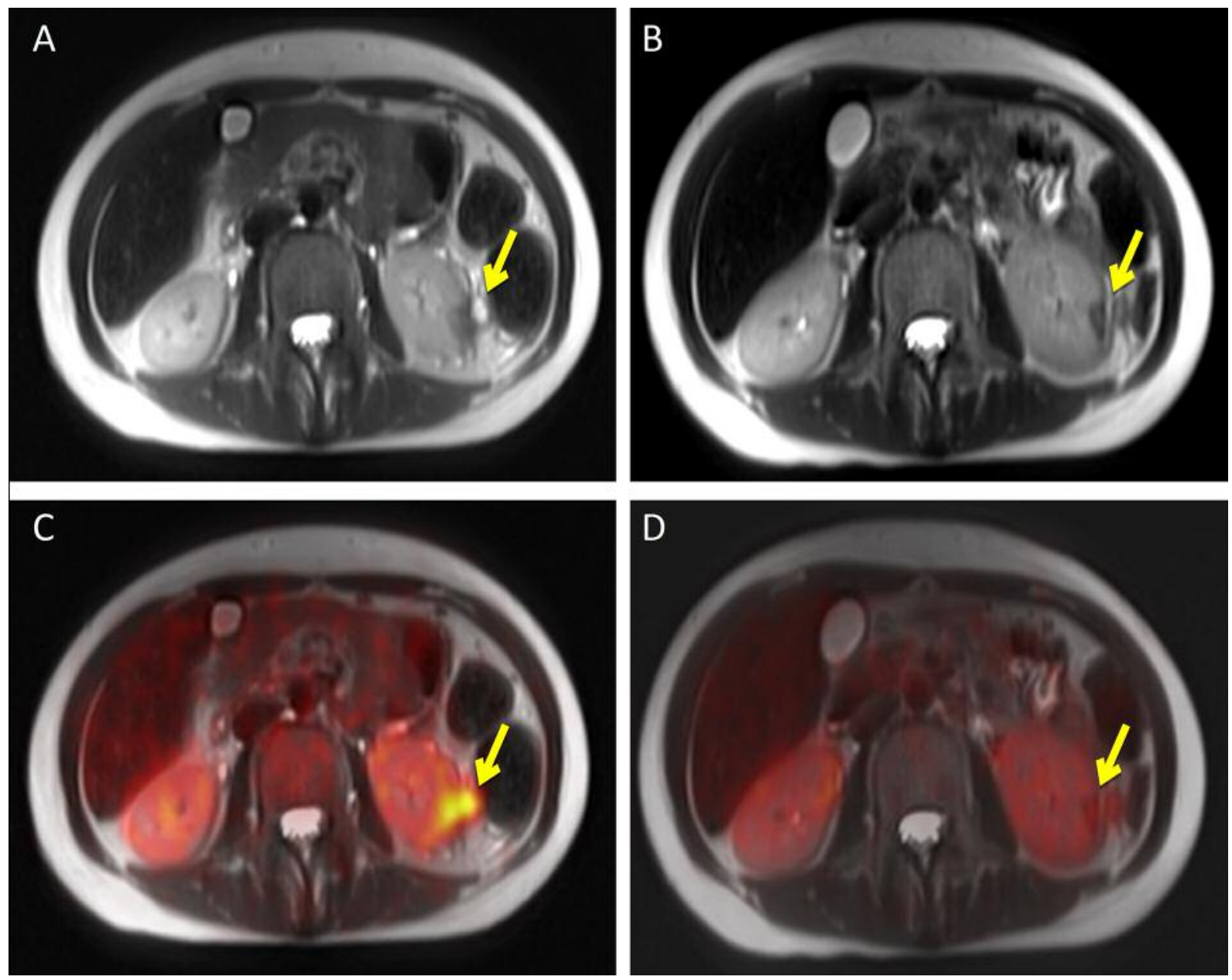

Figure 14. Left kidney Aspergillus lesion (arrows) in a 16-year-old girl on MRI T2-weighted and fused PET/MR images at diagnosis (A and C) and after four weeks of antifungal treatment $(B$ and $D)$ : the lesion shows a slight reduction in size and a significant decrease in metabolic activity. 


\section{Conclusion}

The knowledge of the most common radiological features of IFD is crucial to obtain a correct and early diagnosis. Nevertheless, the differential diagnosis with other infections and with neoplastic lesions can be challenging since fungal infections often do not show specific radiological characteristics; the histological examination is still frequently required when feasible for a definite diagnosis. Whole-body ${ }^{18}$ F-FDG-PET/MRI allows the simultaneous acquisition of both morphological and metabolic information with a lowdose radiation exposure, which represents a significant advantage in comparison with PET-CT especially among the paediatric population. PET/MRI can indeed be considered a useful diagnostic tool in the assessment of disease extent and the evaluation of treatment response in paediatric patients affected by IFD.

\section{Conflicts of Interest}

The Authors declare that no honorarium, grant nor other form of payment was given to anyone to produce the manuscript. The Authors declare that they have no potential conflicts of interest to disclose.

\section{Authors' Contributions}

Varotto A, Orsatti G, Crimì F: review design, data collection, and manuscript drafting; Cecchin D, Toffolutti T: data collection, and manuscript revision; Stramare R, Zucchetta P: review design, and manuscript revision.

\section{References}

1 Ruhnke $\mathrm{M}$ and Schwartz S: Recent developments in the management of invasive fungal infections in patients with oncohematological diseases. Ther Adv Hematol 7(6): 345-359, 2016. PMID: 27904738. DOI: $10.1177 / 2040620716656381$

2 Katragkou A, Fisher BT, Groll AH, Roilides E and Walsh TJ: Diagnostic imaging and invasive fungal diseases in children. J Pediatric Infect Dis Soc 6(suppl_1): S22-S31, 2017. PMID: 28927203. DOI: $10.1093 /$ jpids/pix055

3 Pana ZD, Roilides E, Warris A, Groll AH and Zaoutis T: Epidemiology of invasive fungal disease in children. J Pediatric Infect Dis Soc 6(suppl_1): S3-S11, 2017. PMID: 28927200. DOI: $10.1093 /$ jpids/pix046

4 Sharma P, Mukherjee A, Karunanithi S, Bal C and Kumar R: Potential role of 18F-FDG PET/CT in patients with fungal infections. AJR Am J Roentgenol 203(1): 180-189, 2014. PMID: 24951213. DOI: 10.2214/AJR.13.11712

5 Garnacho-Montero J, Olaechea P, Alvarez-Lerma F, AlvarezRocha L, Blanquer J, Galván B, Rodriguez A, Zaragoza R, Aguado JM, Mensa J, Solé A and Barberán J: Epidemiology, diagnosis and treatment of fungal respiratory infections in the critically ill patient. Rev Esp Quimioter 26(2): 173-188, 2013. PMID: 23817660.
6 Smith JA and Kauffman CA: Pulmonary fungal infections. Respirology 17(6): 913-926, 2012. PMID: 23817660.

7 Smith A, Thimmappa V, Shepherd B, Ray M, Sheyn A and Thompson $\mathrm{J}$ : Invasive fungal sinusitis in the pediatric population: Systematic review with quantitative synthesis of the literature. Int J Pediatr Otorhinolaryngol 90: 231-235, 2016. PMID: 27729140. DOI: 10.1016/j.ijporl.2016.09.019

8 Middlebrooks EH, Frost CJ, De Jesus RO, Massini TC, Schmalfuss IM and Mancuso AA: Acute invasive fungal rhinosinusitis: a comprehensive update of $\mathrm{CT}$ findings and design of an effective diagnostic imaging model. AJNR Am J Neuroradiol 36(8): 1529-1535, 2015. PMID: 25882281. DOI: 10.3174/ajnr.A4298

9 Groppo ER, El-Sayed IH, Aiken AH and Glastonbury CM: Computed tomography and magnetic resonance imaging characteristics of acute invasive fungal sinusitis. Arch Otolaryngol Head Neck Surg 137(10): 1005-1010, 2011. PMID: 22006778. DOI: $10.1001 /$ archoto.2011.170

10 Gavito-Higuera J, Mullins CB, Ramos-Duran L, Olivas Chacon CI, Hakim N and Palacios E: Fungal infections of the central nervous system: a pictorial review. J Clin Imaging Sci 6: 24, 2016. PMID: 27403402. DOI: 10.4103/2156-7514.184244

11 Almutairi BM, Nguyen TB, Jansen GH and Asseri AH: Invasive aspergillosis of the brain: radiologic-pathologic correlation. Radiographics 29(2): 375-379, 2009. PMID: 19325053. DOI: 10.1148/rg.292075143

12 Andes DR, Safdar N, Baddley JW, Playford G, Reboli AC, Rex JH, Sobel JD, Pappas PG, Kullberg BJ and Mycoses Study Group: Impact of treatment strategy on outcomes in patients with candidemia and other forms of invasive candidiasis: a patient-level quantitative review of randomized trials. Clin Infect Dis 54(8): 1110-1122, 2012. PMID: 22412055. DOI: 10.1093/ $\mathrm{cid} / \mathrm{cis} 021$

13 Pastakia B, Shawker TH, Thaler M, O'Leary T and Pizzo PA: Hepatosplenic candidiasis: wheels within wheels. Radiology 166(2): 417-421, 1988. PMID: 3275982. DOI: 10.1148/ radiology.166.2.3275982

14 Metser U, Haider MA, Dill-Macky M, Atri M, Lockwood G and Minden M: Fungal liver infection in immunocompromised patients: depiction with multiphasic contrast-enhanced helical CT. Radiology 235(1): 97-105, 2005. PMID: 15731367. DOI: 10.1148/radiol.2351031210

15 Hot A, Maunoury C, Poiree S, Lanternier F, Viard JP, Loulergue P, Coignard H, Bougnoux ME, Suarez F, Rubio MT, Mahlaoui $\mathrm{N}$, Dupont B, Lecuit M, Faraggi M and Lortholary O: Diagnostic contribution of positron emission tomography with (18F)fluorodeoxyglucose for invasive fungal infections. Clin Microbiol Infect 17(3): 409-417, 2011. PMID: 20636432. DOI: 10.1111/j.1469-0691.2010.03301.x
Received July 22, 2019

Revised August 3, 2019

Accepted August 5, 2019 